\title{
Birds in the west coast of South Kalimantan, Indonesia
}

\author{
MAULANA KHALID RIEFANI ${ }^{1, \boldsymbol{v}}$, MOCHAMAD ARIEF SOENDJOTO ${ }^{2,3, v \vee}$ \\ ${ }^{1}$ Faculty of Teacher Training and Education Sciences, Universitas Lambung Mangkurat. J1. Hasan Basry, Kayutangi, Banjarmasin 70123, South \\ Kalimantan, Indonesia. Tel.: +62-511-3304914, "email: maulanakriefani@ulm.ac.id \\ ${ }^{2}$ Faculty of Forestry, Universitas Lambung Mangkurat. Jl. Ahmad Yani Km 36, Banjarbaru 70714, South Kalimantan, Indonesia \\ Tel.: +62-511-4772290, ${ }^{\text {}}$ email: masoendjoto@ulm.ac.id \\ ${ }^{3}$ Program of Education Biology, Graduate Program, Universitas Lambung Mangkurat. Jl. Hasan Basry, Kayutangi, Banjarmasin 70123, South \\ Kalimantan, Indonesia
}

Manuscript received: 29 November 2020. Revision accepted: 23 December 2020

\begin{abstract}
Riefani MK, Soendjoto MA. 2021. Birds in the west coast of South Kalimantan, Indonesia. Biodiversitas 22: 278-287. There is no comprehensive data on birds in the west coast of South Kalimantan, yet the areas are pressured by human activities, especially the rapidly growing tourism. This study aimed to investigate the diversity of bird species that occurred in the west coast of South Kalimantan and to compare the similarities of bird communities across four villages used as the sampling sites, i.e. Sungai Rasau, Sungai Bakau, Tabanio, and Pagatan Besar. Transect method combined with concentration count method were used to record diurnal bird species and number of individuals in five replications from April to September 2020. The observation area was $100 \mathrm{~m}$ from the shoreline toward the sea and $400 \mathrm{~m}$ from the shoreline toward the inland along $2 \mathrm{~km}$ with observation time was 07.00-11.00 and 16.0019.00. Shannon Wienner species diversity index $\left(\mathrm{H}^{\prime}\right)$, a Dice community similarity index based on the presence or absence of species (ISD), and a community similarity index (modification) based on the number of individuals per species (ISM) were analyzed. In total, there are 101 bird species recorded belonging to 41 families with Sungai Bakau Village has the highest number of species (81 species), while Pagatan Besar Village is the lowest (62 species). Thirty-four species are categorized as waterbirds. Twenty-three species are categorized as migratory birds. In terms of diversity index from highest to lowest are Sungai Bakau (4.04), Sungai Rasau (3.95), Pagatan Besar (3.65), and Tabanio (3.48). The similarity of the ISD community ranges from 0.74-0.84, while the ISM ranges from $0.73-0.90$. One case (Sungai Bakau - Tabanio) shows that community similarity based on the number of individuals per species is smaller than based on the presence or absence of species. Based on the protection status following Indonesian government regulation, most (84.16\%) birds are categorized as unprotected, while based on the conservation status of IUCN, the majority (91.09\%) are Least Concern. The results of this study can be used as baseline information for sustainable management of the area in various aspects, including future research (such as on pest and disease control), tourism (such as bird watching), and education (such as field guide).
\end{abstract}

Keywords: Diurnal bird, diversity, South Kalimantan, similarity, status

\section{INTRODUCTION}

South Kalimantan is one of the five provinces in the main island of Kalimantan (Indonesian Borneo) which has coastlines. The coastlines are located in the southwest, south and east of the province. In more detail, the coastline stretches from the west of the Barito River estuary (administratively included in the Barito Kuala District which borders the Central Kalimantan Province), to the east of the Barito River estuary in Banjar District, to the south to Tanjung Selatan in Tanah Laut District, to the northeast through Tanah Laut District to Pagatan in Tanah Bumbu District, and to the north through Tanah Bumbu District and Kotabaru District to the border with East Kalimantan Province.

Like most coastal ecosystems, the west coast of South Kalimantan also consists of two habitats with different characteristics. The first part is the land/terrestrial habitat, which is located from the shoreline toward the inland. The other part is the beach, which is actually the transition area between land and sea, and is located from the shoreline towards the sea at the lowest tide. The shoreline is a virtual line along the coast which is actually the outermost part of the vegetated land. This line separates the land from the ocean, which is covered by seawater. The beach is alternately or periodically flooded and not inundated by seawater. When high tide or seawater moves inland, beach either covered with vegetation or non-vegetated (sandy/muddy beaches) appears inundated or even submerged. On the other hand, when the sea recedes or the seawater moves away from the land towards the sea, the beach becomes dry and the vegetation that grows or appears to be growing on dry land and non-vegetated areas (sandy/muddy beaches) is evident. According to the Ramsar Convention Secretariat (2016), beach is classified as wetlands. The Ramsar Convention stated that wetlands are areas of marsh, fen, peatland or water, whether natural or artificial, permanent or temporary, with water that is static or flowing, fresh, brackish or salt, including areas of marine water the depth of which at low tide does not exceed six meters.

The west coast of South Kalimantan is an interesting area to study its biological resources, especially the diversity of birds (avifauna). There are two rationales for the importance of studying bird diversity in this region. First, there is no comprehensive information on the species 
richness of bird in this area although preliminary surveys suggested that there were many bird species occurred on the west coast which can be classified into aquatic and nonaquatic birds. The only research is from Riefani and Arsyad (2019) who reported that among the aquatic birds that occurred in Pagatan Besar Village, there were birds that were categorized as migratory birds. Second, four villages on the west coast of South Kalimantan Province have developed tourism, yet the attracting objects have been merely focused on physical resources. The villages of Tabanio, Takisung, and Batakan, for example, make sandy beaches and seawater as tourism features. One other village, namely Pagatan Besar Village, utilizes mangrove forest as a tourist attraction. However, the growth and development of mangroves, especially api-api or white mangrove (Avicennia marina) planted by the community (Soendjoto 2019) hampered activities to observe migratory birds that used to forage on muddy beaches.
This study aimed to investigate the diversity of bird species that occurred in the west coast of South Kalimantan and to compare the similarities of bird communities across four villages used as the sampling sites. We expected the results of this study can be used as baseline information for sustainable management of the area in various aspects, including future research (such as on pest and disease control), tourism (such as bird watching), and education (such as field guide).

\section{MATERIALS AND METHODS}

\section{Study area}

Data were collected from four villages in the west coast of South Kalimantan which were directly adjacent to the Java Sea, namely Sungai Rasau, Sungai Bakau, Tabanio, and Pagatan Besar (Figure 1). Detailed information on geographical site and habitat characteristics are presented in Table 1.

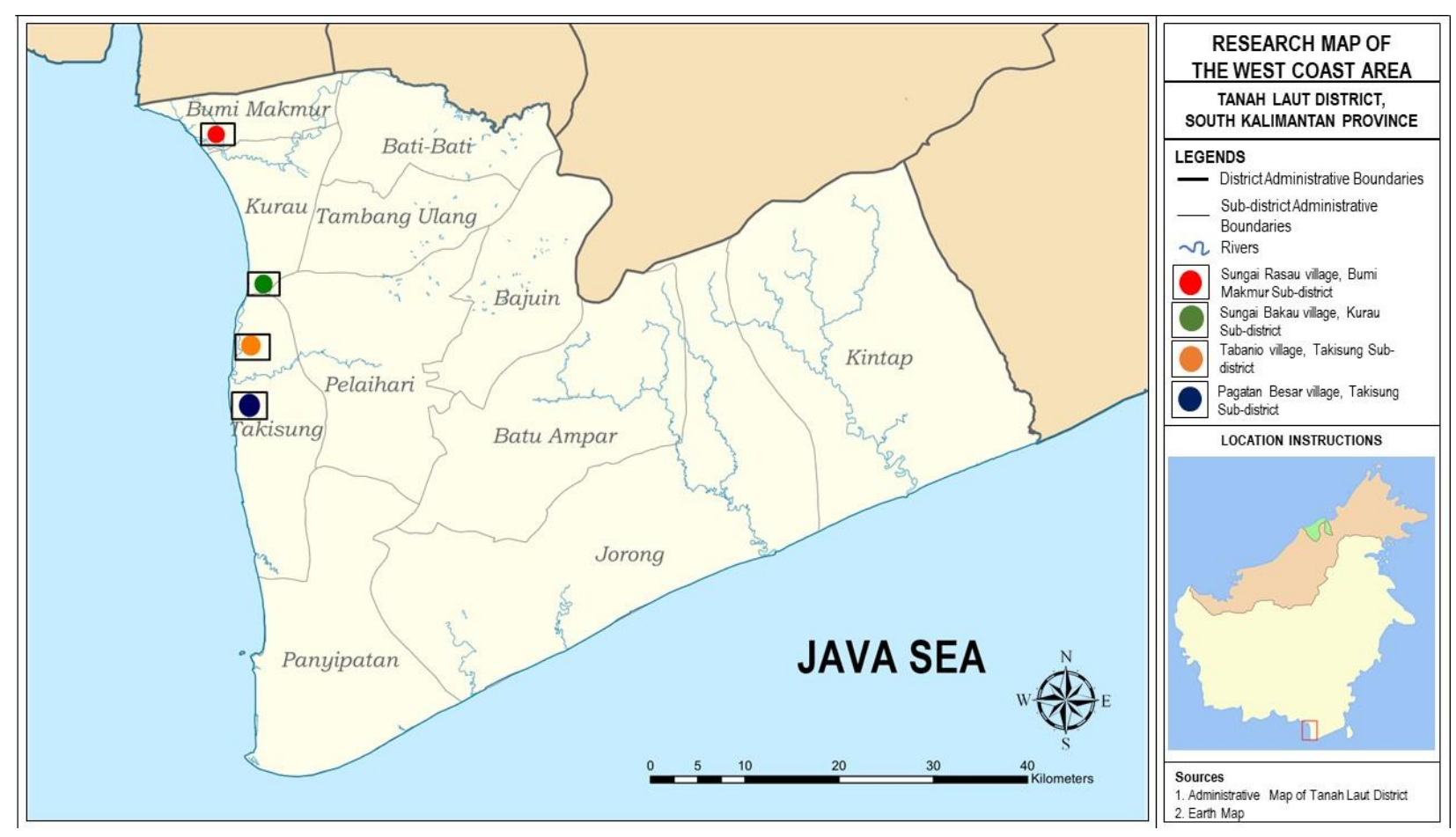

Figure 1. Map of four research sites located in the west coast of South Kalimantan Province, Indonesia

Table 1. Site of data collection, geographical coordinates and habitat characteristics

\begin{tabular}{|c|c|c|c|}
\hline $\begin{array}{l}\text { Site } \\
\text { code }\end{array}$ & Site & Geographical coordinates & Habitat characteristics \\
\hline SR & $\begin{array}{l}\text { Sungai Rasau Village, Bumi Makmur } \\
\text { Sub-district, Tanah Laut District }\end{array}$ & $\begin{array}{l}3^{\circ} 3330^{\prime \prime} \mathrm{S}-3^{\circ} 34^{\prime} 35^{\prime \prime} \mathrm{S} \\
114^{\circ} 34^{\prime} 06^{\prime \prime} \mathrm{E}-114^{\circ} 34^{\prime} 50^{\prime \prime} \mathrm{E}\end{array}$ & $\begin{array}{l}\text { Muddy/sandy flat, mangrove forest, coastal forest, } \\
\text { fishpond, rice field, grass field, settlements }\end{array}$ \\
\hline SB & $\begin{array}{l}\text { Sungai Bakau Village, Kurau Sub- } \\
\text { district, Tanah Laut District }\end{array}$ & $\begin{array}{l}3^{\circ} 42^{\prime} 26^{\prime \prime} \mathrm{S}-3^{\circ} 43^{\prime} 48^{\prime \prime} \mathrm{S} \\
114^{\circ} 37^{\prime} 01^{\prime \prime E}-114^{\circ} 37^{\prime} 34^{\prime \prime} \mathrm{E}\end{array}$ & $\begin{array}{l}\text { Muddy/sandy flat, mangrove forest, coastal forest, } \\
\text { fishpond, rice field, grass field, settlements }\end{array}$ \\
\hline TB & $\begin{array}{l}\text { Tabanio Village, Takisung Sub- } \\
\text { district, Tanah Laut District }\end{array}$ & $\begin{array}{l}3^{\circ} 45^{\prime} 28^{\prime \prime S}-3^{\circ} 46^{\prime} 38^{\prime \prime S} \\
114^{\circ} 36^{\prime} 28^{\prime \prime} \mathrm{E}-114^{\circ} 36^{\prime} 46^{\prime \prime} \mathrm{E}\end{array}$ & $\begin{array}{l}\text { Muddy/sandy flat, mangrove forest, coastal forest, rice } \\
\text { field, grass field, settlements }\end{array}$ \\
\hline PB & $\begin{array}{l}\text { Pagatan Besar Village, Takisung } \\
\text { Sub-district, Tanah Laut District }\end{array}$ & $\begin{array}{l}3^{\circ} 47^{\prime} 56^{\prime \prime} \mathrm{S}-3^{\circ} 49^{\prime} 08^{\prime \prime} \mathrm{S} \\
114^{\circ} 36^{\prime} 14^{\prime \prime} \mathrm{E}-114^{\circ} 36^{\prime} 32^{\prime \prime} \mathrm{E}\end{array}$ & $\begin{array}{l}\text { Muddy flat, mangrove forest, grass field (grass height is } \\
\text { about } 5 \mathrm{~cm} \text {; this meadow is used as a pasture for } \\
\text { livestock), settlements }\end{array}$ \\
\hline
\end{tabular}




\section{Data collection}

Data collection was carried out 5 times between AprilSeptember 2020 using transect and the concentration count methods. The occurrences of diurnal birds were recorded in an area of $100 \mathrm{~m}$ from the shoreline toward the sea and 400 $\mathrm{m}$ from the shoreline toward the inland along $2 \mathrm{~km}$ twice a day at 07.00-10.00 and 16.00-19.00. Along the transect, we walked slowly while identifying species and counting the number of individuals that were seen directly perching or flying on both sides of the transect within a maximum distance of $100 \mathrm{~m}$ if the land was open or $25 \mathrm{~m}$ if the vegetation (trees) was dense. In the concentration count method, we stopped at a certain point (in this case at a densely vegetated or forested area that was thought to be a gathering place or a completely open area where the birds gathered together), observed, identified the species, and counted the number of individuals. The tools used were GPS, binocular $(8 \times 40)$, counter and camera with telephoto lens. Birds were identified according to MacKinnon et al. (2010), Soendjoto et al. (2015), or Soendjoto et al. (2019). The taxonomical name was referred to the IUCN (2020).

\section{Data analysis}

The name of bird species and the number of individuals in five replications were tabulated and counted. Then, species diversity and community similarity indices were calculated using the following formula.

Species diversity index Shannon-Wienner;

$$
\mathrm{H}^{\prime}=-\sum \frac{\mathrm{ni}}{\mathrm{N}} \ln \left(\frac{\mathrm{ni}}{\mathrm{N}}\right)
$$

Where, $\mathrm{ni}=$ number of individuals of species $i ; \mathrm{N}=$ number of individuals of all species; $\ln =$ natural logarithm.

Community similarity index Dice;

$$
\mathrm{IS}_{\mathrm{D}}=\frac{2 \mathrm{~A}}{2 \mathrm{~A}+\mathrm{B}+\mathrm{C}}
$$

Modified community similarity index;

$$
\mathrm{IS}_{\mathrm{M}}=\frac{\mathrm{ni}(\mathrm{A}+\mathrm{B})}{\mathrm{ni}(\mathrm{A}+\mathrm{B})+\mathrm{nj} \mathrm{A}+\mathrm{nkB}}
$$

Where, ISD = Dice community similarity index calculated based on the presence or absence of species; ISM = community similarity index whose equation is a modification of the ISD and the calculation is based on the number of individuals of each species from the communities being compared; $\mathrm{A}=$ number of species present at the two compared sites; $\mathrm{B}=$ number of species present only at the first site; $C=$ number of species present only at the second site; ni $(\mathrm{A}+\mathrm{B})=$ the number of individuals of species $i$ present at site $\mathrm{A}$ and at the same time at site $\mathrm{B} ; \mathrm{nA}=$ number of individuals of species $j$ only at site $\mathrm{A}$; and $\mathrm{nkB}=$ the number of individuals of the $k$ species that exist only at site $\mathrm{B}$.

In addition, the status of the bird species protection was determined according to Regulation of the Minister of Environment and Forestry, Republic of Indonesia No. P.106/MENLHK/SETJEN/KUM.1/12/2018 concerning Amendment to Regulation of the Minister of Environment and Forestry No P.20.MENLHK/SETJEN/KUM.1/6/2018 on The Protected Flora and Fauna, while the conservation status assigned according to the IUCN (2020).

\section{RESULTS AND DISCUSSION}

\section{Species diversity}

In the west coast of South Kalimantan, a total of 101 bird species belonging to 41 families were recorded (Table 2). The village of Sungai Bakau has the richest species with 81 species, Sungai Rasau Village with 78 species, Tabanio Village with 62 species, while Pagatan Besar Village is the lowest with 62 species. The sites with the highest to lowest diversity of bird species are Sungai Bakau (4.04), Sungai Rasau (3.95), Pagatan Besar (3.69), and Tabanio (3.48).

\begin{tabular}{|c|c|c|c|c|c|c|c|c|}
\hline \multirow{2}{*}{ Family/species } & \multirow{2}{*}{ Indonesia name } & \multirow{2}{*}{ Internasional name } & \multicolumn{4}{|c|}{ Number of individuals $\left(n_{i}\right)$} & \multicolumn{2}{|c|}{ Status } \\
\hline & & & SR & SB & TB & PB & P.106 & IUCN \\
\hline \multicolumn{9}{|l|}{ Acanthizidae } \\
\hline Gerygone sulphurea & Remetuk laut & Golden-bellied Gerygone & 21 & 24 & 8 & 15 & TD & LC-d \\
\hline \multicolumn{9}{|l|}{ Accipitridae } \\
\hline Elanus caeruleus & Elang tikus & Black-winged Kite & 5 & 2 & 1 & 1 & $\mathrm{D}$ & LC-s \\
\hline Haliaeetus leucogaster & Elang laut perut putih & White-bellied Sea-eagle & 1 & 2 & 1 & 1 & $\mathrm{D}$ & LC-d \\
\hline Haliastur indus & Elang bondol & Brahminy Kite & 3 & 1 & 1 & 2 & $\mathrm{D}$ & LC-d \\
\hline Nisaetus cirrhatus & Elang brontok & Changeable Hawk-eagle & 1 & - & - & - & $\mathrm{D}$ & LC-i \\
\hline \multicolumn{9}{|l|}{ Acrocephalidae } \\
\hline Acrocephalus orientalis & Kerakbasi besar & Oriental Reed-warbler & 1 & - & - & - & TD & LC-d \\
\hline \multicolumn{9}{|l|}{ Aegithinidae } \\
\hline Aegithina tiphia & Cipoh kacat & Common Iora & 25 & 18 & 14 & 20 & TD & $\mathrm{LC}-\mathrm{u}$ \\
\hline Aegithina viridissima & Cipoh jantung & Green Iora & 4 & 4 & 8 & 6 & TD & NT-d \\
\hline \multicolumn{9}{|l|}{ Alcedinidae } \\
\hline Alcedo meninting & Raja-udang meninting & Blue-eared Kingfisher & 1 & 4 & 1 & 3 & TD & LC-d \\
\hline Halcyon smyrnensis & Cekakak belukar & White-breasted Kingfisher & 4 & 2 & 1 & - & TD & LC-i \\
\hline Pelargopsis capensis & Pekaka emas & Stork-bellied Kingfisher & 3 & 3 & 1 & 4 & TD & LC-d \\
\hline Todiramphus chloris & Cekakak sungai & Collared Kingfisher & 43 & 18 & 28 & 22 & TD & LC-d \\
\hline Todiramphus sanctus & Cekakak suci & Sacred Kingfisher & 6 & 29 & 12 & 7 & TD & LC-i \\
\hline
\end{tabular}

Table 2. Species name, number of individuals and conservation status of bird at the four study sites in the west coast of South Kalimantan, Indonesian 
Anatidae

Anas gibberifrons

Dendrocygna arcuata

Apodidae

Apus nipalensis

Collocalia linchi

Ardeidae

Ardea purpurea

Ardeola speciosa

Butorides striata

Egretta alba

Egretta garzetta

Ixobrychus cinnamomeus

Ixobrychus sinensis

Artamidae

Artamus leucoryn

Campephagidae

Lalage nigra

Pericrocotus igneus

Caprimulgidae

Caprimulgus affinis

Charadriidae

Charadrius dubius

Charadrius leschenaultii

Charadrius mongolus

Pluvialis fulva

Ciconiidae

Leptoptilos javanicus

Cisticolidae

Orthotomus ruficeps

Orthotomus sericeus

Prinia flaviventris

Columbidae

Chalcophaps indica

Geopelia striata

Spilopelia chinensis

Treron vernans

Cuculidae

Cacomantis merulinus

Cacomantis sonneratii

Centropus bengalensis

Centropus sinensis

Chrysococyx minutillus

\section{Dicaeidae}

Dicaeum trigonostigma

Dicaeum trochileum

\section{Estrildidae}

Lonchura fuscans

Lonchura malacca

Lonchura punctulata

Hirundinidae

Delichon dasypus

Hirundo tahitica

Laniidae

Lanius schach

\section{Laridae}

Chlidonias leucopterus

Gelochelidon nilotica

Sterna hirundo

Sterna sumatrana

Sternula albifrons

Thalasseus bergii

Meropidae

Merops philippinus

Merops viridis
Itik benjut

Belibis kembang

Kapinis rumah

Walet linci

Cangak merah

Blekok sawah

Kokokan laut

Kuntul besar

Kuntul kecil

Bambangan merah

Bambangan kuning

Kekep babi

Kapasan kemiri

Sepah tulin

Cabak kota

Cerek kalung-kecil

Cerek-pasir besar

Cerek-pasir mongolia

Cerek krenyut

Bangau tongtong

Cinenen kelabu

Cinenen merah

Prenjak rawa

Delimukan zamrud

Perkutut jawa

Tekukur

Punai gading

Wiwik kelabu

Wiwik lurik

Bubut alang-alang

Bubut besar

Kedasi laut

Cabai bunga api

Cabai jawa

Bondol kalimantan

Bondol rawa

Bondol peking

Layang-layang rumah

Layang-layang batu

Bentet kelabu

Dara laut sayap putih

Dara-laut tiram

Dara-laut biasa

Dara-laut tengkuk-hitam

Dara-laut kecil

Dara-laut jambul

Kirik-kirik laut

Kirik-kirik biru
Sunda Teal

Wandering Whistling-duck

House Swift

Cave Swiftlet

Purple Heron

Javan Pond-heron

Green-backed Heron

Green White Egret

Little Egret

Cinnamon Bittern

Yellow Bittern

White-breasted Woodswallow

Pied Triller

Fiery Minivet

Savanna Nightjar

Little Ringed Plover

Greater Sandplover

Lesser Sandplover

Pacific Golden Plover

Lesser Adjutant

Ashy Tailorbird

Rufous-tailed Tailorbird

Yellow-bellied Prinia

Emerald Dove

Zebra Dove

Eastern Spotted Dove

Pink-necked Green-Pigeon

Plaintive Cuckoo

Banded Bay Cuckoo

Lesser Coucal

Greater Coucal

Little Bronze-cuckoo

Orange-bellied Flowerpecker

Scarlet-headed Flowerpecker

Dusky Munia

Tricoloured Munia

Scally-breasted Munia

Asian House-martin

Tahiti Swallow

Long-tailed Shrike

White-winged Tern

Common Gull-billed Tern

Common Tern

Black-naped Tern

Little Tern

Great Crested Tern

Blue-tailed Bee-eater

Blue-throated Bee-eater
TD NT-s

$\begin{array}{lllll}12 & 15 & - & - & \text { TD }\end{array}$

$\begin{array}{llllll}23 & 20 & 9 & - & \text { TD } & \text { LC-i }\end{array}$

$\begin{array}{llllll}27 & 24 & 21 & 22 & \text { TD } & \text { LC-d }\end{array}$

- 2 - $\quad$ - TD LC-d

$\begin{array}{llllll}24 & 45 & 8 & 9 & \text { TD } & \text { LC-u }\end{array}$

$\begin{array}{llllll}7 & 9 & 1 & 5 & \text { TD } & \text { LC-d }\end{array}$

$\begin{array}{llllll}12 & 4 & - & - & \text { TD } & \text { LC-u }\end{array}$

$\begin{array}{llllll}42 & 10 & 2 & 4 & \text { TD } & \text { LC }-i\end{array}$

$\begin{array}{llllll}4 & 5 & 1 & - & \text { TD } & \text { LC-s }\end{array}$

$\begin{array}{llllll}4 & 8 & 1 & - & \text { TD } & \text { LC }-\mathrm{u}\end{array}$

$\begin{array}{llllll}16 & 14 & 8 & 9 & \text { TD } & \text { LC-s }\end{array}$

$\begin{array}{llllll}8 & 6 & 5 & 12 & \text { TD } & \text { LC-d }\end{array}$

4 - $\quad$ - $\quad$ - TD NT-d

$\begin{array}{llllll}8 & 13 & 5 & 6 & \text { TD } & \text { LC-s }\end{array}$

$\begin{array}{llllll}7 & 62 & - & - & \text { TD } & \text { LC-s }\end{array}$

$9 \quad 33 \quad-\quad 85 \quad$ TD $\quad$ LC-d

- 20 - 20 TD LC-u

- $34 \quad-\quad 27$ TD LC-d

$\begin{array}{llllll}2 & 1 & - & - & \text { D } & \text { VU-d }\end{array}$

$\begin{array}{llllll}23 & 24 & 11 & 16 & \text { TD } & \text { LC-s }\end{array}$

$2 \quad-\quad 2 \quad-\quad$ TD LC-s

$\begin{array}{llllll}8 & 18 & 1 & 6 & \text { TD } & \text { LC-d }\end{array}$

- $\quad$ - 4 - 4 TD LC-d

$\begin{array}{lllll}8 & 1 & 5 & \text { TD } & \text { LC-s }\end{array}$

$\begin{array}{llllll}4 & 20 & 2 & 6 & \text { TD } & \text { LC-i }\end{array}$

$\begin{array}{llllll}4 & 12 & 2 & 6 & \text { TD } & \text { LC-s }\end{array}$

$\begin{array}{llllll}3 & 2 & 3 & 5 & \text { TD } & \text { LC-s }\end{array}$

$\begin{array}{llllll}- & 1 & - & - & \text { TD LC-s }\end{array}$

$\begin{array}{llllll}10 & 10 & 3 & 4 & \text { TD } & \text { LC- } i\end{array}$

$\begin{array}{llllll}3 & 4 & 3 & 4 & \text { TD } & \text { LC-s }\end{array}$

1 - $\quad-\quad-$ TD LC-s

$\begin{array}{llllll}10 & 27 & 6 & 5 & \text { TD } & \text { LC-s }\end{array}$

$\begin{array}{llllll}12 & 18 & 8 & 8 & \text { TD } & \text { LC-s }\end{array}$

$\begin{array}{llllll}39 & 13 & 27 & 10 & \text { TD } & \text { LC-s }\end{array}$

$\begin{array}{llllll}- & - & - & 15 & \text { TD } & \text { LC-1 }\end{array}$

$\begin{array}{llllll}15 & 14 & 11 & 10 & \text { TD } & \text { LC-u }\end{array}$

$\begin{array}{llllll}16 & 20 & 8 & 22 & \text { TD } & \text { LC-u }\end{array}$

$9 \quad 14 \quad-\quad-\quad$ D LC-s

$\begin{array}{llllll}5 & - & - & - & \text { D } & \text { LC-d }\end{array}$

$\begin{array}{llllll}15 & 16 & 15 & 16 & \text { D } & \text { LC-u }\end{array}$

7 - $\quad$ - $\quad-\quad$ D LC-u

- 29 - $\quad-\quad$ D LC-d

- $34 \quad-\quad 12$ D LC-s

$\begin{array}{llllll}9 & 13 & 4 & 8 & \text { TD } & \text { LC-s }\end{array}$

$\begin{array}{llllll}14 & 11 & 31 & 5 & \text { TD } & \text { LC-s }\end{array}$ 
Motacillidae

Anthus novaeseelandiae
Motacilla flava
Muscicapidae
Cyornis rufigastra
Nectariniidae
Aethopyga siparaja
Anthreptes malacensis
Cinnyris jugularis
Leptocoma calcostetha
andionidae

Passeridae

Passer montanus

Pellorneidae

Malacocincla sepiaria

Picidae

Chrysophlegma miniaceum

Pittidae Picoides moluccensis

Pitta sordida

Ploceidae

Ploceus manyar

Pycnonotidae

Pycnonotus aurigaster

Pycnonotus brunneus

Pycnonotus goiavier

Rallidae

Pycnonotus plumosus

Amaurornis cinerea

Amaurornis phoenicurus

Recurvirostridae Himantopus leucocephalus

Rhipiduridae

Rhipidura javanica

Scolopacidae

Actitis hypoleucos

Calidris alba

Calidris ruficollis

Gallinago megala

Limosa lapponica

Numenius madagascariensis

Numenius phaeopus

Tringa glareola

Tringa nebularia

Tringa stagnatilis

Xenus cinereus

Strigidae

Ketupa ketupu

Otus lempiji

\section{Sturnidae}

Acridotheres javanicus

Timaliidae

Mixornis gularis

Vangidae

Hemipus hirundinaceus

Zosteropidae

Zosterops flavus

Zosterops palpebrosus
Apung tanah

Kicuit kerbau

Sikatan bakau

Burung-madu sepah-raja

Burung-madu kelapa

Burung-madu sriganti

Burung-madu bakau

Elang tiram

Burung gereja erasia

Pelanduk semak

Pelatuk merah

Caladi tilik

Paok hijau

Manyar jambul

Cucak kutilang

Merbah mata merah

Merbah cerukcuk

Merbah belukar

Tikusan alis-putih

Kareo padi

Gagang bayam timur

Kipasan belang

Trinil pantai

Kedidi putih

Kedidi leher merah

Berkik rawa

Biru laut ekor-blorok

Gajahan timur

Gajahan penggala

Trinil semak

Trinil kaki-hijau

Trinil rawa

Trinil bedaran

Beluk ketupa

Celepuk reban

Kerak kerbau

Ciung-air coreng

Jinjing batu

Kacamata jawa

Kacamata biasa
Australasian Pipit

Western Yellow Wagtail

Mangrove Blue-flycatcher

Crimson Sunbird

Brown-throated Sunbird

Olive-backed Sunbird

Copper-thorated Sunbird

Osprey

Eurasian Tree Sparrow

Horsfield's Babbler

Banded Woodpecker

Sunda Pygmy Woodpecker

Western Hooded Pitta

Streaked Weaver

Sooty-headed Bulbul

Red-eyed Bulbul

Yellow-vented Bulbul

Olive-winged Bulbul

White-browed Crake

White-brested Waterhen

White-headed Stilt

Sunda Pied Fantail

Common Sandpiper

Sanderling

Rufous-necked Stint

Swinhoe's Snipe

Bar-tailed Godwit

Far Eastern Curlew

Whimbrel

Wood Sandpiper

Common Greenshank

Marsh Sandpiper

Terek sandpiper

Buffy Fish-owl

Sunda Scops-owl

Javan Myna

Pin-striped Tit-babbler

Black-winged Flycatcher-shrike

Javan White-eye

Indian White-eye

Number of individuals (N)

Number of species (S)

Species diversity index $\left(\mathrm{H}^{\prime}\right)$ $\begin{array}{llllll}1 & 3 & 2 & 3 & \text { TD } & \text { LC- }\end{array}$

$\begin{array}{llllll}2 & 2 & 1 & - & \text { TD } & \text { LC-d }\end{array}$

$\begin{array}{llllll}- & - & - & - & & \\ - & - & 1 & 1 & \text { TD }\end{array}$

$\begin{array}{llllll}2 & 3 & 2 & 1 & \text { D } & \text { LC-s }\end{array}$

$\begin{array}{llllll}15 & 12 & 11 & 6 & \text { TD } & \text { LC-s }\end{array}$

$\begin{array}{llllll}13 & 19 & 14 & 4 & \text { TD } & \text { LC-s }\end{array}$

1 - 1 - $\quad$ - TD LC-s

D LC-i

$\begin{array}{llllll}22 & 21 & 41 & 23 & \text { TD } & \text { LC-d }\end{array}$

- $\quad-\quad 2 \quad-\quad$ TD LC-d

- $\quad-\quad 2 \quad-\quad$ TD LC-s

$\begin{array}{llllll}5 & 5 & 1 & 3 & \text { TD } & \text { LC }-\mathrm{i}\end{array}$

- $\quad-\quad 3$ - D LC-d

$2 \quad 10 \quad-\quad-\quad$ TD LC-s

$\begin{array}{llllll}53 & 61 & 76 & 50 & \text { TD } & \text { LC-d }\end{array}$

$\begin{array}{llllll}15 & 4 & 44 & 2 & \text { TD } & \text { LC-d }\end{array}$

$\begin{array}{llllll}15 & 34 & 26 & 37 & \text { TD } & \text { LC-i }\end{array}$

$\begin{array}{cccccc}15 & 34 & 26 & 37 & \text { TD } & \text { LC- } \\ 4 & - & 4 & - & \text { TD } & \text { LC-s }\end{array}$

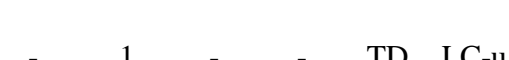

$\begin{array}{llllll}3 & 9 & 3 & 12 & \text { TD } & \text { LC-u }\end{array}$

$\begin{array}{llllll}12 & 8 & - & 3 & \text { TD } & \text { LC }-\mathrm{i}\end{array}$

$\begin{array}{llllll}15 & 11 & 13 & 8 & \text { TD } & \text { LC- }\end{array}$

$\begin{array}{llllll}11 & 12 & 3 & 6 & \text { TD } & \text { LC-d }\end{array}$

- 40 - 5 TD LC-u

- $41 \quad-\quad 18$ TD NT-d

- $\quad-\quad 4 \quad-\quad$ TD LC-u

$\begin{array}{llllll}7 & 13 & - & 8 & \text { TD } & \text { NT-d }\end{array}$

- 4 - 7 D EN-d

- 6 - $\quad$ - D LC-d

$\begin{array}{lllll}5 & 24 & - & & \end{array}$ TD LC-s

- $11 \quad-\quad-\quad$ TD LC-s

$\begin{array}{lllll}5 & 8 & - & - & \text { TD } \\ 10 & 23 & - & 28 & \text { TD }\end{array}$

$\begin{array}{lllll}10 & 23 & - & 28 & \text { TD }\end{array}$

$\begin{array}{llll}- & - & - & \text { TD LC-s }\end{array}$

$\begin{array}{llllll}- & - & 1 & - & \text { TD } & \text { LC-s }\end{array}$

$\begin{array}{llllll}25 & 55 & 25 & 7 & \text { TD } & \text { VU-d }\end{array}$

$\begin{array}{llllll}15 & 35 & 7 & 13 & \text { TD } & \text { LC-s }\end{array}$

$\begin{array}{lllll}- & 4 & - & 1 & \text { TD }\end{array}$

3 - $\quad-\quad$ - $\quad$ TD EN-d

$\begin{array}{llllll}2 & 8 & - & - & \text { TD } & \text { LC-d }\end{array}$

$\begin{array}{llll}817 & 1242 & 576 & 705\end{array}$

$\begin{array}{llll}78 & 81 & 63 & 62\end{array}$

$\begin{array}{llll}3.95 & 4.04 & 3.48 & 3.69\end{array}$

Note: 1) Site of data collection: SR: Sungai Rasau, SB: Sungai Bakau, TB: Tabanio, PB: Pagatan Besar. 2) Protection status according to Minister of Environment and Forestry, Republic of Indonesia No P.106/MENLHK/SETJEN/KUM.1/12/2018 (2018): D: protected, TD: unprotected. 3) Conservation status according to IUCN (2020): EN: endangered, VU: vulnerable, NT: near threatened, LC: least concern, i: increasing, d: decreasing, s: stable, u: unknown 
Most (66.34\% or 67 species) of the 101 bird species are categorized as non-waterbirds, while the rest $(34.66 \%)$ are waterbirds. Eight waterbird families in the west coast include of Anatidae, Ardeidae, Charadriidae, Ciconiidae, Laridae, Rallidae, Recurvirostridae, and Scolopacidae, and are among 34 families of waterbirds in the world described by Ducks Unlimited New Zealand (2017) or among 33 families described by Wetlands International (2020).

Twenty-one of 34 waterbird species are categorized as migratory birds, including Greater Sandplover, Lesser Sandplover, Pacific Golden Plover, Little Ringed Plover, Common Gull-billed Tern, Little Tern, White-winged Tern, Great Crested Tern, Common Tern, White-headed Stilt, Far Eastern Curlew, Marsh Sandpiper, Whimbrel, Common Sandpiper, Terek Sandpiper, Wood Sandpiper, Common Greenshank, Swinhoe's Snipe, Sanderling, Rufous-necked Stint, and Bar-tailed Godwit. Based on the colored flag attached to the bird's leg and referring to the meaning of the flag color in EAAFP (2018), R. Yuliansyah (2020, pers. com.) found Sanderling of South Australia on the sandy stretch of the Sungai Bakau, and T.K. Utomo (2020, pers. com.) found Rufous-necked Stint of China-Chongming Island in Pagatan Besar.

Besides the migratory waterbirds, there are also nonwaterbirds that are categorized as migrants. They are Osprey, the raptor and Blue-tailed Bee-eater, the insectivore. Osprey has been observed soaring above waters and ponds and then perching on the dry tree of the Sungai Rasau. The presence of Osprey at that site indicates that the migratory raptors (Germi et al. 2009, Bierregaard et al. 2014, Purwanto et al. 2015) or these wetland raptors (Irham 2012) make the west coast as a stopover site. In the southern hemisphere, these birds that spread around the world become non-breeding visitors in Indonesia (van Balen 1998, Purwanto et al. 2015) as well as breeding visitors in Australia (Clancy 2006; Dennis and Clancy 2014, Detmar and Dennis 2018). Osprey was recorded in February-March 2016 flying around an artificial lake in the Tarjun Cement Factory Area, Kotabaru (Riefani et al. 2019).

Blue-tailed Bee-eater and Blue-throated Bee-eater are migratory birds in Malaysia (Nisbet 2013) and Thailand (DeCandido et al. 2010). There has not been any specific publication on the bee-eaters as migratory birds in Indonesia. According to Soendjoto et al. (2018) and Riefani et al. (2019), Blue-tailed Bee-eater was not always found, while Blue-throated Bee-eater was almost always found in 2013-2017 observations in Tabalong District, South Kalimantan and more than 300 hours of field observation in Kotabaru District, South Kalimantan. Therefore, we only include Blue-tailed Bee-eater as migratory birds and not Blue-throated Bee-eater. Nash and Nash (1985) mentioned Blue-tailed Bee-eater as one of the migratory birds in South Sumatra and do not mention Blue-throated Bee-eater as such.

The following two bird species were also found on the west coast. Both are highlighted in this paper because their presence and distribution are not stated in field books, such as Smythies (1960) and MacKinnon et al. (2010). Therefore, these findings can be considered as new records that are important for updating data or revising field books. The first is White-breasted Kingfisher which was found in Tanah Laut District, to be precise at Sungai Rasau, Sungai Bakau, and Tabanio. The presence of the bird was observed 9 times, both solitary and 2 individuals, adding to new information that this bird is indeed distributed in Kalimantan Selatan or even Kalimantan in general. Smythies (1960) did not mention the presence of this bird in Borneo. Avibase (2020b) even said that not a single subspecies of White-breasted Kingfisher existed in Indonesia. According to Avibase (2020b), the birds spreading across the Arabian Peninsula to the Caucasus Mountains and the northwestern part of India are Halcyon smyrnensis smyrnensis; in West India and Sri Lanka H.s. fusca, in the Andaman H.s. saturatior; as well as in Myanmar to Peninsular Malaysia and Indochina H.s. perpulchra. MacKinnon et al. (2010) stated that the distribution of this bird in Sumatra and Java. According to Strange (2012), its distribution was even only in Sumatra. The distribution of White-breasted Kingfisher in Sumatra is supported by the report of Ayat (2011), Arief et al. (2015), Kamal et al. (2016), Santosa et al. (2016), Syamsi (2017), and Iswandaru et al. (2018), while in Java there was the report by Taufiqurrahman et al. (2019). White-breasted Kingfisher was also found in West and South Kalimantan (Myers 2016), industrial plantation companies in West Kalimantan (Ata Marie and Ekologika Consultans 2017), Sebangau National Park, Central Kalimantan (Adi 2018), oil palm plantations in Central Kalimantan (Santosa et al. 2018), and the Liang Anggang Protected Forest Area, Kalimantan Selatan (Wulansari et al. 2020).

The second is Streaked Weaver which was found breeding in Sungai Rasau and Sungai Bakau. In the observations conducted in July 2020, an average of 7.3 nests hung per coconut tree (Cocos nucifera) $(\mathrm{n}=10)$ at about $20 \mathrm{~m}$ above ground level. More nests (up to 32 nests) were found hanging from branches of the Acacia auriculiformis tree at about 6-10 $\mathrm{m}$ above ground level. The tree is on the roadside of Sungai Bakau Village. The distribution of Streaked Weaver in Tanah Laut District adds to new record in primary publications. Early records suggest that the distribution of Streaked Weaver in Indonesia is only in Java and Bali (MacKinnon et al. 2010) and not recorded in Borneo (Smythies 1960, MacKinnon et al. 2010). IUCN (2020) said that this bird spreads in Southeast Asia (Cambodia, Myanmar, Singapore, Thailand, Vietnam), South Asia (India, Bangladesh, Bhutan, Nepal, Pakistan, Sri Lanka), China, and even Egypt. Particularly in Indonesia, the map attached to the IUCN report (2020) shows that the distribution of Streaked Weaver is consistent with MacKinnon et al. (2010) mentioned above. According to Avibase (2020a), the Streaked Weaver spreading across Indonesia (the islands of Java, Bali and Bawean) is a subspecies of Ploceus manyar manyar; in eastern Pakistan to western India and Sri Lanka P.m. flaviceps; in Northeast India (Assam) to Bangladesh and northern Myanmar P.m. peguensis; and in Southwest China (Yunnan) to Thailand and Vietnam P.m. williamsoni.

The presence of Streaked Weaver in Kalimantan was published by Soendjoto et al. (2014) and Iqbal et al. (2016). 
The nests hung from the branches of rambai or mangrove apple (Sonneratia caseolaris) and rumbia or sago palm (Metroxylon sago) trees. From interviews with the community, Soendjoto et al. (2016) stated that this bird had been detected in Banjar District in 2011. It is likely that the birds were brought by bird traders from Java Island and then accidentally released. Birds can also be deliberately released by keepers who are tired of raising animals or are not careful with raising them after buying them from traders.

In a field trip in 2017, we even found 3 occurrences of this bird with its nest hanging individually on bundung or greater club rush (Cyperaceae: Scirpus grossus), one of the swamp plants around Sungai Puting, Tapin Distcrit. On each stem of 1-1.5 m high there was only one nest attached. In 2018 the weaver's nest was found hanging on a branch of the mangrove apple at a height of about $20 \mathrm{~m}$ above the ground in the swamplands of Lambung Mangkurat University, Banjarmasin City. Streaked Weaver is considered to be pests, along with Dusky Munia, Dusky Munia, Scally-breasted Munia, and Eurasian Tree Sparrow because they eat rice grown by the people living in the settlements west of the university campus complex.

It is important to note that the states of the bird species richness and diversity indices are temporary (subject to change) and only apply to this study due to the following reasons. First, this study focuses on diurnal birds, so that nocturnal birds are not observed and identified. Observation and identification of only certain diurnal bird species, for example, those that are small in size and inhabit mangrove forests, coastal forests, or forests with thick canopy cover or those that are migrants, require a longer duration or frequency of observation. Observation and identification of nocturnal bird species are relatively challenging. Certain strategies or techniques need to be developed because what is faced is not only the dark sight, but also the thickness of the canopy and/or tidal inundation. In this study, two nocturnal bird species that were found by chance were Buffy Fish-owl and Sunda Scops-owl. It is likely that there are other nocturnal bird species at the sites.

Second, one sub-district on the west coast (Panyipatan sub-district) has not been represented in the study. However, the following findings of bird species from a wildlife photographer can be used as a guide that there are actually other birds on the west coast. Lesser Frigatebird (Fregata andrewsi), Great Tit (Parus major), Black-bellied Malkoha (Phaenicophaeus diardi), Buff-banded Rail (Hypotaenidia philippensis), and Copper-throated Sunbird (Nectarinia calcostetha) were sighted in the area by $\mathrm{P}$. Irawan (2020, pers. com.).

\section{Community similarity}

The community similarity index between sites based on the presence or absence of species $(0.77-0.84)$ is lower than that based on the number of individuals per species $(0.79$ 0.90) (Table 3). The exception is the index based on the presence or absence of species between Tabanio and the
Sungai Bakau which is slightly higher than that based on the number of individuals of each species. Since the index based on the number of individuals for each species is generally higher or closer to 1.00 , this index is better to use to measure community similarity between two sites.

The similar resources with adequate availability are the factors that cause the similarity of bird communities in the two compared sites to be categorized as high. These resources include food, nesting, microclimate and occurrence of predators. In the case of migratory bird species, both intra and inter continents, birds leave certain sites and come to other sites because they have the same resource. Site and season cause the availability of prey and species requirements for energy to vary and in turn the proportion of foraging birds also varies (Burton et al. 2004). The presence and diversity of migratory birds in various habitat types is largely determined by microhabitat, availability of food sources, and safety in resting and foraging areas (Sonobe and Usui 1993, Howes et al. 2003).

In the Sungai Bakau, the feed source area is disturbed by tidal inundation. Some waterbirds, or to be precise in this case are shorebirds, generally forage on mudflats that lie after the shoreline towards the sea. They forage in colonies within the same species and even with other waterbird species. However, when the high tide inundates or submerges the muddy areas, they move to and forage on other muddy areas, such as active ponds, non-operational ponds or former ponds. In this non-vegetated inland muddy field, they can still stand on the surface of the mud and their beaks can still reach the food on the surface or in the mud. Moving and looking for food in the mud inland is a strategy for waterbirds to survive. In general, the waterbird diet consists of plants, such as terrestrial plants, seeds and aquatic macrophytes, and animals, such as zooplankton, snails, shells, worms, crabs, mollusks, crustaceans, arthropods, larvae, and imago of terrestrial and aquatic insects (Coleoptera, Diptera, Heteroptera, Odonata), nectonic macroinvertebrates, fish, amphibians, and water snakes (Ntiamoa-Baidu et al. 1998, Horváth et al. 2012). The feed is obtained easily when the mud bed is not inundated by seawater and facilitates the birds to behave according to their morphology; for example, the soles and toes may step on the mud and the beak reaches for feed in the mud.

Table 3. Bird community similarity index between sites

\begin{tabular}{lcccc}
\hline Site & $\begin{array}{c}\text { Sungai } \\
\text { Rasau }\end{array}$ & $\begin{array}{c}\text { Sungai } \\
\text { Bakau }\end{array}$ & Tabanio & $\begin{array}{c}\text { Pagatan } \\
\text { Besar }\end{array}$ \\
\hline Sungai Rasau & 1.00 & 0.87 & 0.90 & 0.85 \\
Sungai Bakau & 0.84 & 1.00 & 0.73 & 0.88 \\
Tabanio & 0.78 & 0.74 & 1.00 & 0.79 \\
Pagatan Besar & 0.77 & 0.84 & 0.78 & 1.00 \\
\hline
\end{tabular}

Note: The numbers below the diagonal line (top left - bottom right) are the community similarity index based on the presence or absence of species, and while above the diagonal line are that based on the number of individuals of each species. 
The conditions that make water birds move are not only the tidal mud area, but also the growth and development of mangrove species. In Pagatan Besar village, for example, the muddy area which was originally an open area has gradually turned into a mangrove forest. The development of mangrove forests makes it difficult for migratory birds who are accustomed to foraging for food together (in colonies) on muddy plains in open areas. This change was observed from four ironwood walkways, which according to Soendjoto (2019) have $1.5 \mathrm{~m}$ wide and about $100 \mathrm{~m}$ long, jutting from the shoreline towards the Java Sea. The height of the walkway floor is $0 \mathrm{~m}$ from the ground level at the starting point of the walkway (at the shoreline) and about $2 \mathrm{~m}$ from the ground level (mud) at the end of the walkway (towards the sea). At Titian 1, which is located at the northernmost point, mangrove forests which are dominated by white mangrove are observed to be getting higher. The height in 2018 was around 4-5 m and in 2020 it is about $6 \mathrm{~m}$. However, the sea and the mud can still be observed from the end of the walkway. At Titian 2, which is located $490 \mathrm{~m}$ to the south of Titian 1, the sea and mud can still be observed from half the length of the walkway to the end of the path in 2018. In 2020, the sea and mud can no longer be observed. The white mangroves reach 3-4 m in high and are covering the view. At Titian 3, which is located $190 \mathrm{~m}$ to the south of Titian 2, the sea and mud can be observed from the base to the end of the bridge in 2018 because the height of the white mangroves growing on the left and right is only about $0.5 \mathrm{~m}$ from the surface of the mud. In 2020, the height of the flames is $2 \mathrm{~m}$ around the base of the walkway and about $1 \mathrm{~m}$ around the end of the walkway. Several individual white mangroves had grown out of the direction of the sea from the end of the bridge. In other words, mangrove forests are growing or expanding. The sea and mudflats can still be observed starting from one-third of the length of the walkway to the end of the path. Titian 4 which is located $180 \mathrm{~m}$ to the south from Titian 3, the sea and mud can still be observed freely. The white mangroves are still few in number. In other words, the density is very low compared to the density of the white mangroves around Titian 1, 2, and 3. The height is also about $0.5 \mathrm{~m}$ above the ground (mud).

The stretch of mud on the west coast is formed by sediments carried by the Maluka River, Tabanio River, Pagatan Besar River, and Barito River as the widest river in South Kalimantan and then deposited in the Java Sea, where these rivers flow into. The main sediments on the Barito River are sand, clay and silt (Arisanty et al. 2014). Because the Barito River is the main route for coal transportation (Novico and Priohandono 2012), coal shale is also observed in the mud. According to Arisanty et al. (2012), shorelines can change and the changes are caused by human activities, such as logging, forest and land fires, intensive agriculture, and mining.

\section{Bird status}

Based on protection status of the regulation of Minister of Environment and Forestry, Republic of Indonesia No P.106/MENLHK/SETJEN/KUM.1/12/2018 (2018), most bird species $(84.16 \%)$ are categorized as unprotected. Only
16 species (15.84\%) are protected. Based on their conservation status of IUCN (2020), most bird species $(91.09 \%)$ are categorized as Least Concern with a tendency of population to decrease (30 species), increase (13 species), stable (36 species), and unknown (13 species). The rest are categorized as Near Threatened (5 species, namely Green Iora, Sunda Teal, Fiery Minivet, Rufousnecked Stint, and Bar-tailed Godwit), Vulnerable (2 species, namely Lesser Adjutant and Javan Myna), and Endangered (2 species, namely Far Eastern Curlew and Javan White-eye. A large number of unprotected bird species while at the same time the least concern status is of course alarming. Many bird species have not been studied intensively, but their sustainability or survival is threatened.

Currently, people catch birds for at least two purposes. First, birds are used as a pet. Zebra Dove, Eastern Spotted Dove, Sooty-headed Bulbul, Yellow-vented Bulbul, and Javan Myna are kept because of their voice. Unfortunately, birds are kept without partners so they cannot reproduce. Second, bird species are consumed. White-breasted Waterhen, Yellow Bittern, and Wandering Whistling-duck are species that are traded and then enjoyed for their meat. The over catching and consumption of birds has definitely wiped them out.

In addition, land conversion, especially the protected areas or conservation areas into production areas also threatens the bird. Forest-dwelling birds who like lush bushes or under-canopy dwellers such as Western Hooded Pitta, Ioras, Pin-striped Tit-babbler, and Emerald Dove have lost their habitats. In contrast, birds that are able to adapt to open areas or grass vegetation find new habitats. They include Eurasian Tree Sparrow, Australasian Pipit, Western Yellow Wagtail, and Javan Myna.

Each bird species has its own ecological roles and functions. As carnivores, eagles and Brahminy Kite help maintain the balance of nature. Sooty-headed Bulbul, Yellow-vented Bulbul, and flowerpeckers are frugivores that help spread plant seeds from certain sites to other sites, so do Zebra Dove, Eastern Spotted Dove, and munias as granivores. Long-tailed Shrike, bee-eaters, and Sunda Pied Fantail as insectivores play a role in controlling pests and diseases in plants. Sunbirds as nectarivores not only act as nectar eaters, but also helps pollinate.

In conclusion, 101 bird species found in the west coast of South Kalimantan are classified as minimal. The species diversity index of the four sample sites ranges from 3.48 to 4.04. In general, the similarity index based on the number of individuals per species is greater than that based on the presence or absence of the species. Migratory birds can be developed as unique attractions or ecotourism objects on this west coast. White-breasted Kingfisher and Streaked Weaver are worth noting in field books when these birds have been found spreading in Kalimantan.

\section{ACKNOWLEDGEMENTS}

This research was funded by PNBP of Lambung Mangkurat University, Banjarmasin, Indonesia in the Fiscal 
Year of 2020. We express our gratitude to the Rector of Lambung Mangkurat University who approved the funding for this research. Thanks also go to the Head of Tanah Laut District, Indonesia who allowed the research to be carried out in his administrative area and the enumeration team (Nur Abdi Suga) who helped collect data in the field.

\section{REFERENCES}

Adi. 2018. Bird Species Identification in Sungai Kaki and Sebangau Kecil. https://www.tnsebangau.com/kegiatan-identifikasi-jenisburung-di-sungai-kaki-dan-sebangau-kecil. [Indonesian]

Arief H, Mijiarto J, Rahman A. 2015. Diversity and protection status of wildlife in PT Riau Sawitindo Abadi. Media Konservasi 20 (1): 159165. DOI: 10.29244/medkon.20.2.\%25p [Indonesian].

Arisanty D, Sartohadi J, Marfai MA, Hadmoko DS. 2012. The Long-term morphodynamic of Barito Delta, Southern Kalimantan, Indonesia. J Environ Sci Eng B 1: 1196-1202.

Arisanty D, Sartohadi J, Marfai MA, Hadmoko DS. 2014. Sediment dynamic in Barito Delta, Southern Kalimantan, Indonesia. J Environ 1 (1): 30-37.

Ata Marie and Ekologika Consultans. 2017. Summary Report High Conservation Value Assessment PT Hutan Ketapang Industri Kabupaten Ketapang, West Kalimantan Province. Ata Marie \& Ekologika Consultans, Jakarta.

Avibase. 2020a. Streaked Weaver Ploceus manyar (Horsfield, 1821). https: //avibase.bsc-eoc.org

Avibase. 2020b. White-throated Kingfisher Halcyon smyrnensis (Linnaeus, 1758). https: //avibase.bsc-eoc.org

Ayat A. 2011. Agroforest Birds in Sumatra. World Agroforestry CentreICRAF, SEA Regional Office, Bogor, Indonesia. [Indonesian]

Bierregaard RO, Poole AF, Washburn BE. 2014. Ospreys (Pandion haliaetus) in the $21^{\text {st }}$ century: Populations, migration, management, and research priorities. J Raptor Res 48 (4): 301-308. DOI: 10.3356/0892-1016-48.4.301

Burton NHK, Musgrove AJ, Rehfisch MM. 2004. Tidal variation in numbers of waterbirds: how frequently should birds be counted to detect change and do low tide counts provide a realistic average? Bird Stud 51: 48-57.

Clancy GP. 2006. The breeding biology of the Osprey Pandion haliaetus on the North Coast of New South Wales. Corella. J Austr Bird Stud Assoc 30 (1): 1-8.

Decandido R, Nualsri C, Allen D. 2010. Mass northbound migration of Blue-tailed Merops philippinus and Blue-throated M. viridis Beeeaters in southern Thailand, spring 2007-2008. Forktail 26: 42-48.

Dennis TE, Clancy GP. 2014. The status of the Osprey (Pandion haliaetus cristatus) in Australia. J Raptor Res 48 (4): 1-7. DOI: 10.3356/JRR OSPR-14-01.1

Detmar SA, Dennis TE. 2018. A review of Osprey distribution and population stability in South Australia. South Australian Ornithologist (43): $1-2$.

Ducks Unlimited New Zealand. 2017. What are waterbirds? https //ducks.org.nz.

EAAFP. 2018. Shorebird Color Flagging Protocol on the East AsianAustralasian Flyway. https: //eaaflyway.net/wpcontent/uploads/2017/12/Protocol_birds-marking.pdf

Germi F, Young GS, Salim A, Pangimangen W, Schellekens M. 2009. Over-ocean raptor migration in a monsoon regime: spring and autumn 2007 on Sangihe, North Sulawesi, Indonesia. Forktail 25: 104-116.

Horváth Z, Ferenczi M, Móra A, Vad CF, Ambrus A, Forró L, Szövényi G, Andrikovics S. 2012. Invertebrate food sources for waterbirds provided by the reconstructed wetland of Nyirkai-Hanyy, northewestern Hungary. Hydrobiologia 697: 59-72. DOI 10.1007/s10750-012-1170-5.

Howes J, Bakewell D, Noor YR. 2003. Panduan Studi Burung Pantai. Wetlands International Indonesia Program, Bogor. [Indonesian]

Iqbal M, Irawan P, Fauzi A, Rahman I. 2016. Introduced Species: Streaked Weaver Ploceus manyar, a new species for Kalimantan, Borneo. Birding ASIA 25: 71-72.

Irham M. 2012. Avifauna diversity at Central Halmahera North Maluku, Indonesia. Zoo Indonesia 21 (1): 17-31.
Iswandaru D, Khalil ARA, Kuniawan B, Pramana R, Febryano IG, Winarno GD. 2018. Abundance and diversity of bird species in Mangrove Forest KPHL Gunung Balak. Indonesian J Conserv 7 (1): 57-62. [Indonesian]

IUCN [International Union for Conservation of Nature]. 2020. The IUCN Red List of Threatened Species. Version 2020-2. https: //www.iucnredlist.org

Kamal S, Agustina E, Rahmi Z. 2016. Bird species in some habitat types in Kecamatan Lhoknga, Kabupaten Aceh Besar. Jurnal Biotik 4 (1): 15-32. [Indonesian]

MacKinnon J, Phillipps K, van Balen B. 2010. LIPI- Field Guide of Birds in Sumatra, Kalimantan, Java and Bali. Puslitbang Biologi- LIPI, Bogor. [Indonesian]

Myers S. 2016. Helm Field Guides, Birds of Borneo, $2^{\text {nd }}$ ed. NHBS, UK.

Nash SV, Nash AD. 1985. A checklist of the forest and forest edge birds of the Padang-Sugihan Wildlife Reserve, South Sumatra. Kukila 2 (3): 51-59.

Nisbet ICT. 2013. Seasonal occurrence, migrations and habitat relationships of Blue-tailed and Blue-throated Bee-eaters Merops philippinus and M. viridis in Peninsular Malaysia. Raffles Bull Zool Suppl. 29: 219-223.

Novico F, Priohandono YA. 2012. Analysis of erosion and sedimentation patterns using software of Mike 21 HDFM-MT in The Kapuas Murung River Mouth Central Kalimantan Province. Bull Mar Geol 27 (1): 35-53.

Ntiamoa-Baidu Y, Piersma T, Wiersma P, Poot M, Battley P, Gordon C. 1998. Water depth selection, daily feeding routines and diets of waterbirds in coastal lagoons in Ghana. Ibis 140 (1): 89-103. DOI: 10.1111/j.1474-919X.1998.tb04545.x

Purwanto AA, Rakhman Z, Supriatna AA, Sutito ASB, Srirejeki I. 2015. Current information on migratory raptors and its conservation efforts in Indonesia. Asian Raptors 1: 54-62.

Rachman R, Sudiarty N, Ismawati, Sumbawati NK, Hun S. 2020. Analysis of household income in swiftlet cultivation in Karang Dima Village, Labuhan Badas District. Prosiding Seminar Nasional IPPeMas 2020 Inovasi Hasil Penelitian dan Pengabdian kepada Masyarakat dalam Menunjang Era Industri 4.0. [Indonesian]

Ramsar Convention Secretariat. 2016. An Introduction to the Convention on Wetlands (previously The Ramsar Convention Manual). Ramsar Convention Secretariat, Gland, Switzerland.

Riefani MK, Arsyad M. 2019. Bird species in Mangrove Ecotourism Area of Pagatan Besar, Tanah Laut Regency, Indonesia. Prosiding Seminar Nasional Lingkungan Lahan Basah 4 (1): 192-196. [Indonesian]

Riefani MK, Soendjoto MA, Munir AM. 2019. Bird species in the cement factory complex of Tarjun, South Kalimantan, Indonesia. Biodiversitas 20 (1): 218-225. DOI: 10.13057/biodiv/d200125

Santosa RA, Harianto SP, Nircahyani N. 2016. Comparison of the population of Kingfisher bird (Halcyonidae) in the wetlands of Sungai Luar Village and the wetlands of Kibang Pacing Village, Menggala Timur District, Tulang Bawang Regency. Jurnal Sylva Lestari 4 (2): 79-88. DOI: 10.23960/js12479-88. [Indonesian]

Santosa Y, Sugiharti W, Erniwati. 2018. The variation of bird diversity in different land cover at oil palm plantation: Case study at Asm Oil Palm Estate in Central Kalimantan, Indonesia. E3S Web of Conferences 52, 00051. DOI: 10.1051/e3sconf/20185200051.

Smythies BE. 1960. The Birds of Borneo. The Sabah Society \& The Malaya Nature Society, Kuala Lumpur, Malaysia.

Soendjoto MA, Nugroho Y, Suyanto, Riefani MK, Supandi, Yudha HES. 2019. Avifauna in the Area of PT Borneo Indobara Kalimantan Selatan. Banyubening, Banjarbaru. [Indonesian]

Soendjoto MA, Riefani MK, Ready A. 2014. New record: Streaked Weaver (Ploceus manyar) in Kalimantan Selatan. Warta Konservasi Lahan Basah 22 (3): 16-17. [Indonesian]

Soendjoto MA, Riefani MK, Triwibowo D, Metasari D. 2018. Birds observed during the monitoring period of 2013-2017 in the revegetation area of ex-coal mining sites in South Kalimantan, Indonesia. Biodiversitas 19 (1): 323-329. DOI: 10.13057/biodiv/d190144

Soendjoto MA, Riefani MK, Triwibowo D, Wahyudi F. 2015. Avifauna in Reclamation Area of PT Adaro Indonesia. Universitas Lambung Mangkurat Press, Banjarbaru, Indonesia. [Indonesian]

Soendjoto MA. 2019. Potential and problems in development of the ecotourism area (Case in the Pagatan Besar Mangrove Forest, Tanah Laut Regency, Indonesia). Prosiding Seminar Nasional Lingkungan Lahan Basah 4 (3): 635-642. 
Sonobe K, Usui S. 1993. A Field Guide to the Waterbirds of Asia. Wild Bird Society of Japan, Tokyo.

Strange M. 2012. A Photographic Guide to the Birds of Indonesia. $2^{\text {nd }}$ ed. Tuttle Publishing, Tokyo.

Sudiyanto T. 2018. The impact of the swiftlet business on changes in the economy of the Betung Banyuasin community. Jurnal Media Wahana Ekonomika 12 (3): 101-109. [Indonesian]

Syamsi F. 2017. Bird diversity in wetland area of Kota Batam. Simbiosa 6 (1): 17-30. [Indonesian]

Taufiqurrahman I, Purwanto AA, Tirtaningtyas FN, Ade S, Rachman M, Daryana A, Hermawan AS, Yordan K, Emmanuel B, Kristanto A, Kiryono, Purnama S, Sujatmiko DA. 2019. Recent records of White- breasted Kingfisher Halcyon smyrnensis on Java, Indonesia, with notes on its status and distribution. BirdingASIA 31: 74-78.

van Balen SB. 1998. Tropical forest raptors in Indonesia: Recent information on distribution, status, and conservation. J Raptor Res 32 (1): 56-63.

Wetlands International. 2020. Waterbird Population Estimates. https: //wpe.wetlands.org.

Wulansari A, Soendjoto MA, Suyanto. 2020. Bird species diversity in natural vegetation of Liang Anggang Protected Forest Area- Block I, Banjarbaru, Indonesia. Jurnal Sylva Scienteae 3 (5): 818-824. [Indonesian] 